\title{
Linguistic Cues Enhance the Learning of Perceptual Cues
}

\author{
Hanako Yoshida and Linda B. Smith \\ Indiana University
}

\begin{abstract}
When language is correlated with regularities in the world, does it enhance the learning of these regularities? This question lies at the core of both notions of linguistic bootstrapping in children and the Whorfian hypothesis. Support for an affirmative answer is provided in an artificial-nounlearning task in which 2-year-old children were taught to distinguish categories of solid and nonsolid things with and without supporting correlated linguistic cues.
\end{abstract}

One common notion is that language serves a bootstrapping function, helping children discover categories (e.g., Brown, 1956; Gleitman, Gleitman, Landau, \& Wanner, 1988; Landau, 1994; Macnamara, 1994; Waxman \& Markow, 1995). Language's effects could be universal and not dependent on the specific language being learned, or they could depend on linguistic devices particular to specific languages. In this article, we identify one bootstrapping mechanism that could create language-specific concepts. As Whorf (1956) proposed, languages differ in the way they correlate with regularities in the world, and in so doing, they may causally influence what one notices and learns, and thus the concepts one has.

The experiment we report here concerns how correlated linguistic cues help children discover the regularities that characterize early noun categories. One regularity is this: The concrete nouns that children know divide (albeit imperfectly) into two subgroups, solid things in shape-based categories and nonsolid things in material-based categories (Samuelson \& Smith, 1999). Thus, among these nouns, solidity predicts category structure. By age 3, children know this. When shown a single novel exemplar and told its name, 3year-olds systematically generalize that name to new instances on the basis of shape for solids but on the basis of material for nonsolids (Imai \& Gentner, 1997; Samuelson \& Smith, 1999; Soja, 1992; Soja, Carey, \& Spelke, 1991; Subrahmanyam, Landau, \& Gelman, 1999).

Younger children do not so consistently exploit these regularities. Whereas the shape bias for solids is a robust phenomenon even in 2-year-olds (Graham, Williams, \& Huber, 1999; Landau, Smith, \& Jones, 1988, 1998), the material bias for nonsolids is much less reliable. In some studies, 2-year-olds have been reported to extend names for nonsolids on the basis of material (Soja, 1992; Soja et al., 1991), but in other studies, 2-year-olds have either overgeneralized the shape bias for solids to nonsolids (Samuelson, 2002; Samuelson \& Smith, 1999) or responded at chance levels (Imai \& Gentner, 1997). Altogether, the evidence suggests that children's knowledge of the predictive relation between solidity and category structure increases between 2 and 3 years of age.

There is also a second regularity in the early English lexicon: Count syntax correlates with solidity and shape-based categories, and mass syntax correlates with nonsolidity and 
material-based categories (Samuelson \& Smith, 1999). In English, then, linguistic cues that are correlated with perceptual cues to solidity also predict category structure. Children learning English are sensitive to these correlations. Count syntax (e.g., "This is a mel") heightens attention to shape, whereas mass syntax (e.g., "This is some mel") heightens attention to material (Dickinson, 1988; Gathercole, Cramer, Somerville, \& Jansen, 1995; Soja, 1992).

The experimental question for this report is how the presence of correlated linguistic cues to category structure influences learning about perceptual cues. Research with adults shows that clusters of correlated cues mutually reinforce each other, leading to stronger individual links than if those links had not been part of an intercorrelated cluster (e.g., Billman, 1996; Billman \& Knutson, 1996; Goldstone, 1998; Medin, Altom, Edelson, \& Freko, 1982; O'Reilly, 2001; see also Yoshida \& Smith, 2003). Figure 1 schematically illustrates the phenomenon: The learned connection between $a$ and $b$ is stronger if acquired in the context of $c$, which correlates with both $a$ and $b$, than if acquired without that redundant correlation. Thus, if children learn about perceptual cues to category structure in the context of a redundant linguistic contrast, the perceptual cues may later be more potent even when the correlated linguistic cues are absent.

In this experiment, we tested this hypothesis by manipulating the presence or absence of a redundant linguistic contrast during learning about perceptual cues to category structure. The participants were young children learning Japanese as their first and only language. We chose child subjects because the impact of correlated linguistic cues may be most potentand most consequential - as young learners form their initial categories. We chose Japanesespeaking children because the Japanese language makes no syntactic distinction analogous to the count-mass distinction in English and no syntactic distinction that correlates with categories of solid versus nonsolid things (Imai \& Gentner, 1997; Lucy, 1992). Finally, early-learned Japanese nouns show the same regularities as those evident among earlylearned English nouns (Colunga \& Smith, in press); that is, solidity is correlated with categories organized by shape, and nonsolidity is correlated with categories organized by material. Thus, in Japanese-speaking children, we have an "experimental preparation" in which the perceptual regularities are the same as in English-speaking children but to which we can experimentally add or not add correlated linguistic cues.

In the experiment, 2-year-old Japanese-speaking children were taught novel names for solids in shape-based categories and novel names for nonsolids in material-based categories. This situation mimics children's learning of specific lexical categories, categories that by hypothesis are the bases for children's knowledge that solid things are named by shape and nonsolid things are named by material. After training, we tested for generalized knowledge in a transfer task using entirely novel names labeling novel solid and nonsolid things.

\section{METHOD}

\section{Participants}

Thirty-six monolingual Japanese-speaking children (mean age $=28.3$ months) were tested in Niigata and in Osaka, Japan. The children were randomly assigned to one of four conditions resulting from crossing the presence or absence of correlated linguistic cues during training with the presence or absence of correlated linguistic cues during testing.

\section{Training}

Children were taught four lexical categories through presentations of two exemplars for each category. Two of these lexical categories referred to pairs of objects that matched only in shape, and two referred to pairs of substances that matched only in material. Samples of 
solid and nonsolid training sets are shown in Figure 2. The four novel words were modo, torode, tego, and narima. Training consisted of a 5-min play period with each lexical category. During each 30-min session, the experimenter simply named the instances in each training pair at least 20 times. For children in the training condition with the correlated linguistic cues, every time a training instance was named, either hitotsu no (for solid exemplars) or sukoshi no (for nonsolid exemplars) was used. For the remaining children, those in the training condition without the correlated linguistic cues, the solid and nonsolid training instances were named using the same sentence frame. The Japanese sentence frames used in training and their English glosses are given in Table 1.

The cues we used, hitotsu and sukoshi, are real Japanese words, lexical items within a complex system of classifiers and quantifiers. We chose these terms because they were syntactically appropriate but unlikely to be known by the children. Hitotsu signifies one discrete inanimate thing. Sukoshi means "a small portion" and is used for continuous substances (e.g., a portion of sand), for a small number of discrete entities (e.g., a few coins), and as a degree modifier (e.g., slightly cold). Neither of these terms nor any other classifier in Japanese is specific to solid or nonsolids (Uchida \& Imai, 1999). Further, hitotsu and sukoshi are terms not commonly used when talking about everyday objects and substances (Naka, 1999). Finally, previous research indicates that Japanese-speaking children younger than 3 years have little understanding of how even more common classifiers relate to different kinds (Matsumoto, 1985, 1986; Yamamoto \& Keil, 2000; see also Naka, 1999; Uchida \& Imai, 1999).

Each child participated in 10 training sessions distributed over a maximum of 4 weeks, with all sessions at least 2 days apart. The order of training pairs was randomly determined at each session for each child.

\section{Test}

Immediately after the completion of the last training session, each child participated in the test phase, which had two conditions, one in which the test questions contained correlated linguistic cues and one in which the questions did not have these cues. The Japanese phrases used in testing and their English glosses are given in Table 2.

On each trial, the child was shown a novel entity (one not used in training), told its name (a novel name not used in training), and then asked to pick from three alternatives another entity with the same name. The key question was whether the children would generalize the name for a novel solid thing on the basis of shape but generalize the name for a novel nonsolid thing on the basis of material. Figure 3 shows two of the test sets. Each test set included the exemplar that was named and three choice objects, each of which matched the exemplar in shape, material, or color. The solid sets were made of materials such as wood, hardened clay, plastic, and metal, and the nonsolid sets were made of materials such as foam, toothpaste, gel, and cream. In total, there were six unique test sets, either solid or nonsolid. Each was repeated three times, for a total of 18 randomly ordered test trials. The novel names used during testing were kochi, taroma, soe, nochira, teto, and hamoku.

\section{Control}

Prior to the main experiment, we conducted a preliminary control experiment. Eight 2-yearold monolingual Japanese-speaking children were tested with the correlated linguistic cues; that is, the novel name was embedded in a sentence frame with hitotsu if the exemplar was solid and was embedded in a sentence frame with sukoshi if the exemplar was nonsolid. There was no prior training. Children performed at chance, generalizing by shape for solid items and by material for nonsolid items on $42 \%$ of the trials (chance $=33 \%$ ), $t(7)<1.89, p$ 
$>.10$; more specifically, they generalized the names for both solids (78\%) and nonsolids (68\%) by shape, $t(7)=0.822, p<.50$. Thus, without training, 2-year-old Japanese-speaking children overgeneralize the shape bias for solids to nonsolids. Further, without training, the two correlated linguistic cues (hitotsu, sukoshi) do not differentially cue attention to shape and material.

\section{RESULTS}

Children's responses on the test trials were scored as correct if the children generalized names for solids on the basis of shape and names for nonsolids on the basis of material. Children who were trained with the correlated linguistic cues outperformed those who were not so trained, both when the linguistic cues were present at test $(.81$ vs. .52 correct $), t(18)=$ $3.86, p<.001$, one-tailed, and when the linguistic cues were not present at test (.64 vs. .48 correct), $t(18)=1.96, p<.05$, one-tailed, $d=0.78$. The second comparison is the crucial one: Learning a correlation between perceptual cues and category structure in the context of a redundant linguistic cue led to enhanced performance even when the correlated linguistic cues were not present at test.

Table 3 provides a more complete accounting of the children's performance. As is evident, children in all conditions extended the names for solids on the basis of shape. In contrast, performance with nonsolids depended on the training condition. A 2 (with or without correlated linguistic cues at training $) \times 2$ (with or without correlated linguistic cues at test) $\times$ 2 (solidity) mixed-design analysis of variance of correct choices revealed a main effect of training condition, $F(1,34)=16.203, p<.001, R^{2}=.41$; a main effect of solidity, $F(1,34)=$ $55.62, p<.001, R^{2}=.704$; and a reliable interaction between solidity and training condition, $F(1,34)=7.28, p<.02, R^{2}=.079$. The main effect of presence of correlated linguistic cues at test was marginally reliable, $F(1,34)=3.68, p<.07$. The interaction between training condition and test condition, $F(1,34)=1.467, p=.23$ was not significant, nor was the interaction among solidity, training condition, and test condition, $F(1,34)=2.41, p=.3$. Post hoc comparisons (Tukey's $H S D, a=.05$ ) again confirmed the critical role of training with the linguistic cues: Children who were trained with redundant linguistic cues extended names for nonsolids on the basis of material more than children who did not receive those cues during training, both when the cues were present at test (.77 vs. .28) and, more crucially, when they were absent (.46 vs. .24). Further, only children trained with correlated linguistic cues showed material-based extensions for nonsolids at levels reliably greater than chance (.33), $t(7)=6.25, p<.01$ (cues present at test) and $t(7)=3.16, p<.01$ (cues absent at test).

In contrast to the children in the preliminary control study, children in all training conditions treated solids and nonsolids differently. This indicates that in all conditions, children learned something from the training. Although correlated linguistic cues may bolster learning about perceptual correlations, they do not appear necessary to that learning. Still, the presence of correlated linguistic cues during training bolstered children's learning of the predictive link between nonsolidity and category structure, and this was evident even when those linguistic cues are not present at test.

\section{DISCUSSION}

One remarkable fact about early lexical learning is how good children are at it, seeming to learn a whole category from hearing a single thing named. Children do this by exploiting cues to category structure - by learning, for example, that solidity predicts shape-based categories, and nonsolidity predicts material-based categories. The present results document a strong role for language in this learning. The addition of redundantly correlated linguistic 
cues reinforces children's learning of the links between perceptual cues and category structure. This effect was predicted by studies and models of adult category learning. In implicit learning tasks, adding a redundant correlation increases the strength of other associations (e.g., Billman \& Knutson, 1996). The present results add to this finding by showing that redundant linguistic cues bolster learning about perceptual cues and by demonstrating that such overlapping correlations do real developmental work, helping children discover and exploit regularities in the learning environment.

Thus, the mechanism behind at least one form of linguistic bootstrapping appears to be the same as that behind the learning of any arbitrary set of associations. In the present experiment, however, the correlated linguistic cues were not truly arbitrary, but rather were real words with potentially relevant meanings for Japanese-speaking adults, although probably not for the young children who participated in the experiment. Rather, for them, the words may have gained meaning through their associations in the training task. Crucially, the experiment shows that by teaching associations between words and perceptual properties, one will change not only what is known about the words, but also what is known about the correlations among the perceptual properties. This is intriguing because languages offer many kinds of devices that redundantly correlate with regularities in the world, and in these ways may broadly influence what is learned about those regularities.

Languages also differ in the redundant correlations they add to the learning environment. If language serves as a bootstrap to category learning, then different languages provide different bootstraps. This does not mean that differences between languages will always lead to dramatic differences in conceptual outcomes. Even without special training, Japanesespeaking children do acquire a solidity-nonsolidity distinction (Imai \& Gentner, 1997). This fact shows that redundant linguistic cues are not necessary to learn about perceptual regularities. Still, languages selectively add redundancies to the regularities in the world, and as we have shown here, those redundancies strengthen learning about the regularities with which they are correlated, and they do so in a way that persists even when the correlated linguistic cues are removed. Thus, it seems that Whorf's original idea about how language shapes categories might be right after all. At the very least, a mechanism that could produce such an effect exists.

\section{Acknowledgments}

This research was supported by a National Institute of Mental Health grant (R01 MH60200) and by Indiana University. We thank the teachers, children, and parents at Yoshida Nursery School in Niigata, Japan, and at Umemoto Nursery School in Osaka, Japan.

\section{REFERENCES}

Billman, D. Structural biases in concept learning: Influences from multiple functions. In: Medin, DL., editor. The psychology of learning and motivation: Advances in research and theory. Vol. Vol. 35. San Diego, CA: Academic Press; 1996. p. 283-321.

Billman D, Knutson J. Unsupervised concept learning and value systematicity: A complex whole aids learning the parts. Journal of Experimental Psychology: Learning, Memory, and Cognition. 1996; 22:458-475.

Brown, R. The original word game. In: Bruner, JS.; Goodnow, JJ.; Austin, GA., editors. A study of thinking. New York: Wiley; 1956. p. 247-312.

Colunga E, Smith LB. A connectionist account of the object-substance distinction in early noun learning. Psychological Review. (in press).

Dickinson DK. Learning names for material: Factors constraining and limiting hypothesis about word meaning. Cognitive Development. 1988; 3:15-35. 
Gathercole VCM, Cramer LJ, Somerville SC, Jansen M. Ontological categories and function: Acquisition of new names. Cognitive Development. 1995; 10:225-251.

Gleitman, LR.; Gleitman, H.; Landau, B.; Wanner, E. Where learning begins: Initial representations for language learning. In: Newmeyer, FJ., editor. Linguistics: The Cambridge Survey: Vol. 3. Language: Psychological and biological aspects. New York: Cambridge University Press; 1988. p. 150-193.

Goldstone RL. Perceptual learning. Annual Review of Psychology. 1998; 49:585-612.

Graham SA, Williams LD, Huber JF. Preschoolers' and adults' reliance on object shape and object function for lexical extension. Journal of Experimental Child Psychology. 1999; 74:128-151. [PubMed: 10479398]

Imai M, Gentner D. A cross-linguistic study of early word meaning: Universal ontology and linguistic influence. Cognition. 1997; 62:169-200. [PubMed: 9141906]

Landau, B. Object shape, object name, and object kind: Representation and development. In: Medin, DL., editor. The psychology of learning and motivation: Advances in research and theory. Vol. Vol. 31. San Diego, CA: Academic Press; 1994. p. 253-304.

Landau B, Smith LB, Jones S. The importance of shape in early lexical learning. Cognitive Development. 1988; 3:299-321.

Landau B, Smith LB, Jones S. Object perception and object naming in early development. Trends in Cognitive Sciences. 1998; 2:19-24. [PubMed: 21244958]

Lucy, JA. Language diversity and thought: A reformulation of the linguistic relativity hypothesis. Cambridge, England: Cambridge University Press; 1992.

Macnamara, J. Logic and cognition. In: Macnamara, J.; Reyes, GE., editors. The logical foundations of cognition. Vol. Vol. 4. New York: Oxford University Press; 1994. p. 11-34.Vancouver Studies in Cognitive Science

Matsumoto Y. A sort of speech act qualification in Japanese: Chotto. Journal of Asian Culture. 1985; 9:142-159.

Matsumoto Y. The Japanese classifier -hon: A prototype-semantic analysis. Sophia Linguistica. 1986; 20/21:73-81.

Medin DL, Altom MW, Edelson SM, Freko D. Correlated symptoms and simulated medical classification. Journal of Experimental Psychology: Learning, Memory, and Cognition. 1982; 8:37-50.

Naka M. The acquisition of Japanese numerical classifiers by 2-4-year-old children: The role of caretakers' linguistic inputs. Japanese Psychological Research. 1999; 41(1):70-78.

O'Reilly RC. Generalization in interactive networks: The benefits of inhibitory competition and Hebbian learning. Neural Computation. 2001; 13:1199-1241. [PubMed: 11387044]

Samuelson LK. Statistical regularities in vocabulary guide language acquisition in connectionist models and 15-20-month-olds. Developmental Psychology. 2002; 38:1016-1037. [PubMed: 12428712]

Samuelson LK, Smith LB. Early noun vocabularies: Do ontology, category structure, and syntax correspond? Cognition. 1999; 73:1-33. [PubMed: 10536222]

Soja N. Inferences about the meanings of nouns: The relationship between perception and syntax. Cognitive Development. 1992; 7:29-46.

Soja N, Carey S, Spelke E. Ontological categories guide young children's inductions of word meanings: Object terms and substance terms. Cognition. 1991; 38:179-211. [PubMed: 2049905]

Subrahmanyam K, Landau B, Gelman R. Shape, material, and syntax: Interacting forces in children's learning in novel words for objects and substances. Language and Cognitive Processes. 1999; 14:249-281.

Uchida N, Imai M. Heuristics in learning classifiers: The acquisition of the classifier system and its implications for the nature of lexical acquisition. Japanese Psychological Research. 1999; 41(1): 50-69.

Waxman SR, Markow DB. Words as invitations to form categories: Evidence from 12-month-old infants. Cognitive Psychology. 1995; 29:257-302. [PubMed: 8556847] 
Whorf, B. Language, thought and reality: Selected writings of Benjamin Lee Whorf. Carroll, JB., editor. Cambridge, MA: MIT Press; 1956.

Yamamoto K, Keil FC. The acquisition of Japanese numerical classifiers-linkages between grammatical forms and conceptual categories. Journal of East Asian Linguistics. 2000; 9:379-409.

Yoshida H, Smith LB. Shifting ontological boundaries: How Japanese- and English-speaking children generalize names for animals and artifacts. Developmental Science. 2003; 6:1-34. 


\section{Training}

Test

\section{No Redundant Correlation}
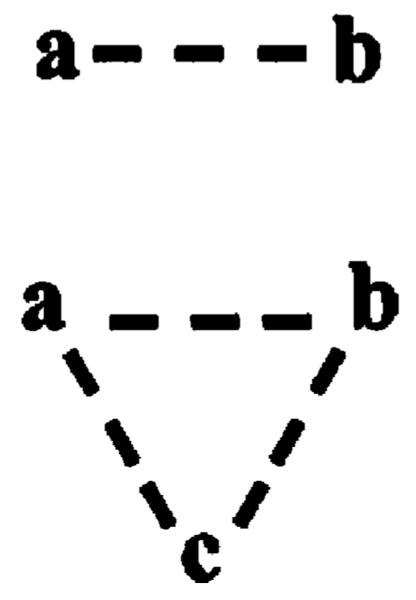

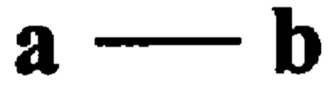

a

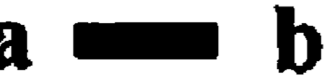

Fig. 1.

Schematic illustration of the interactive effects of redundantly correlated cues. The correlations that exist before training (dashed lines) are strengthened after training (solid lines). The thicker line indicates greater expected strength. 


\section{Solid}
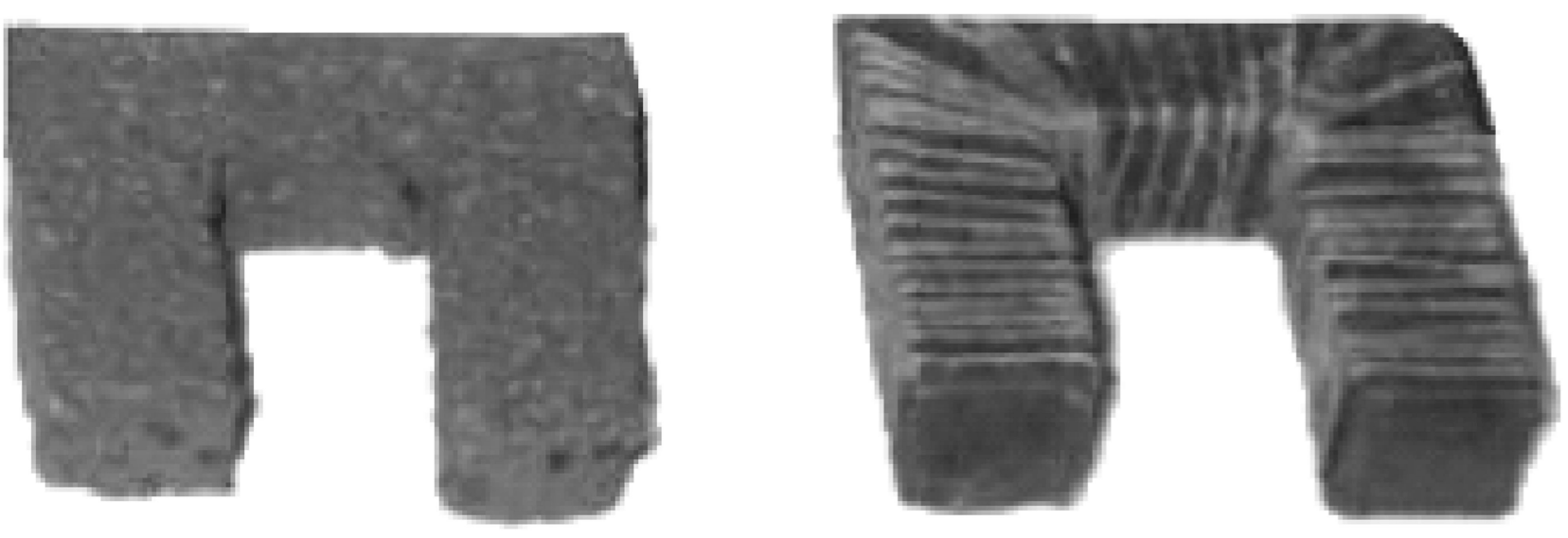

Nonsolid
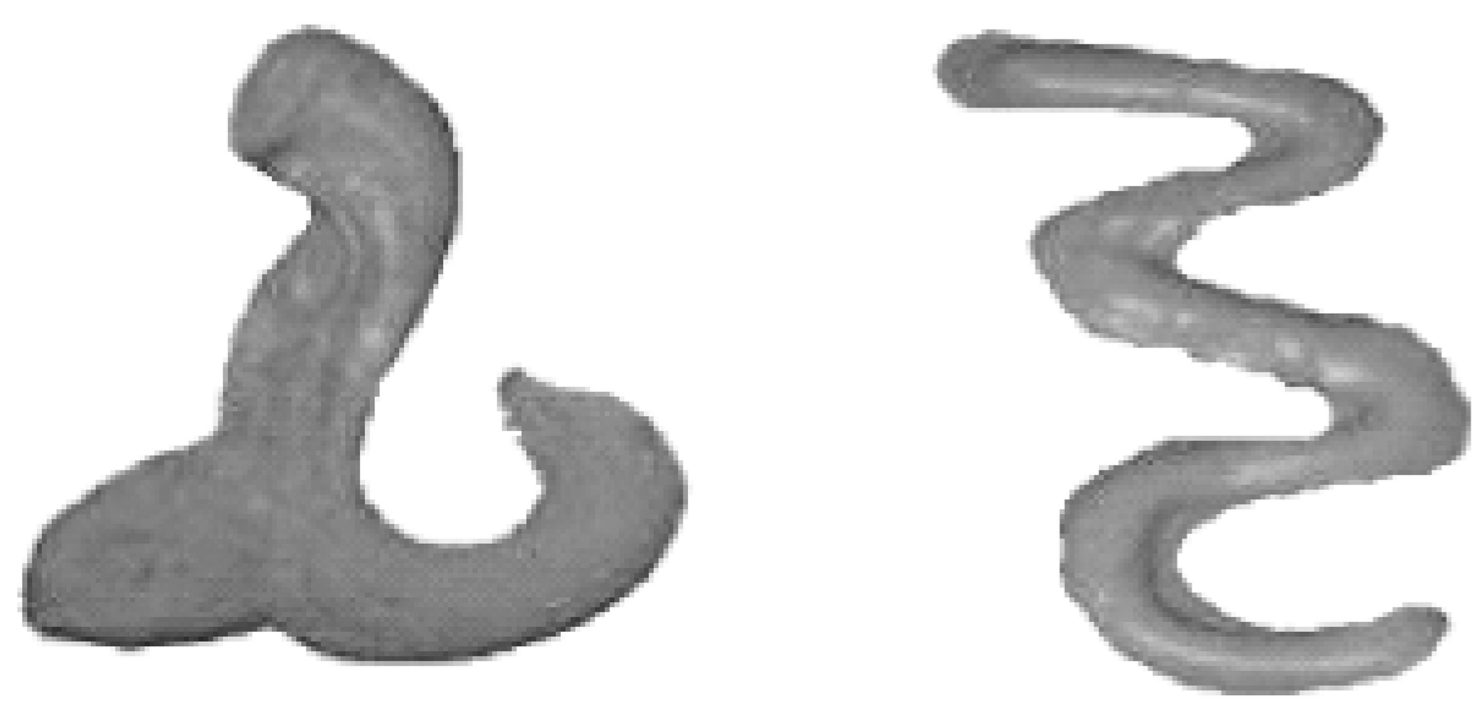

Fig. 2.

Samples of solid and nonsolid stimulus pairs used for the training sessions. 


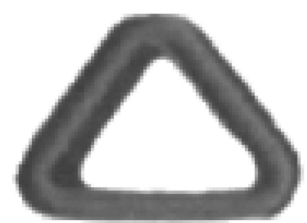

Shape Choice

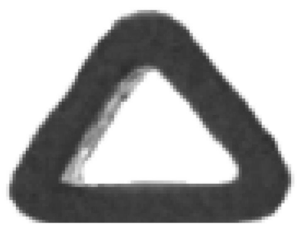

\section{Solid Exemplar}

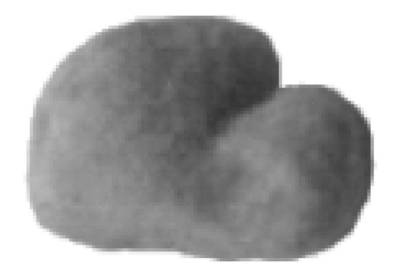

Material Choice

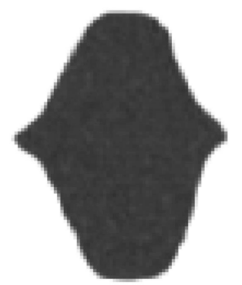

Color Choice

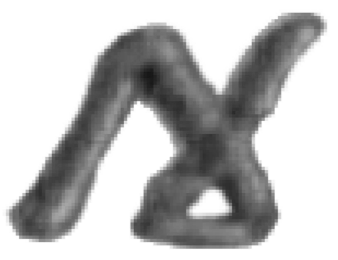

Nonsolid Exemplar

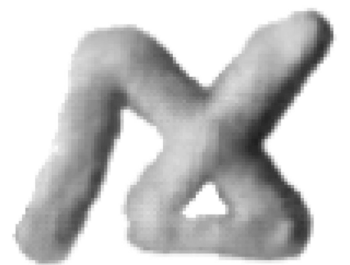

Shape Choice

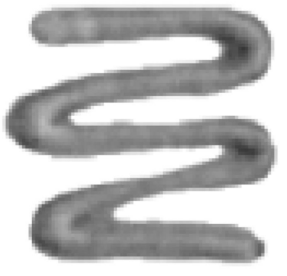

Material Choice

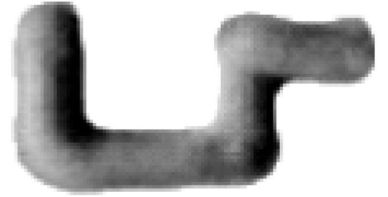

\section{Color Choice}

Fig. 3.

Samples of the test sets. Each set included a solid or nonsolid exemplar and a choice of three stimulus items that matched the exemplar in shape, material, or color. 
TABLE 1

The Japanese Sentence Frames Used in Training

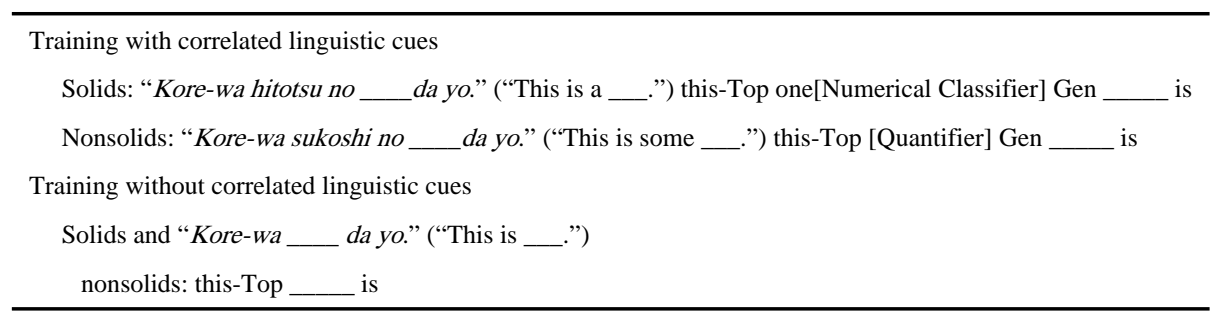

Note. Japanese has postpositional particles to mark syntactic and semantic roles. The postpositional particles in these sentences are the topic marker (-Top), wa, and the genitive case marker (-Gen), no. 
TABLE 2

The Japanese Sentence Frames Used in the Test Phase

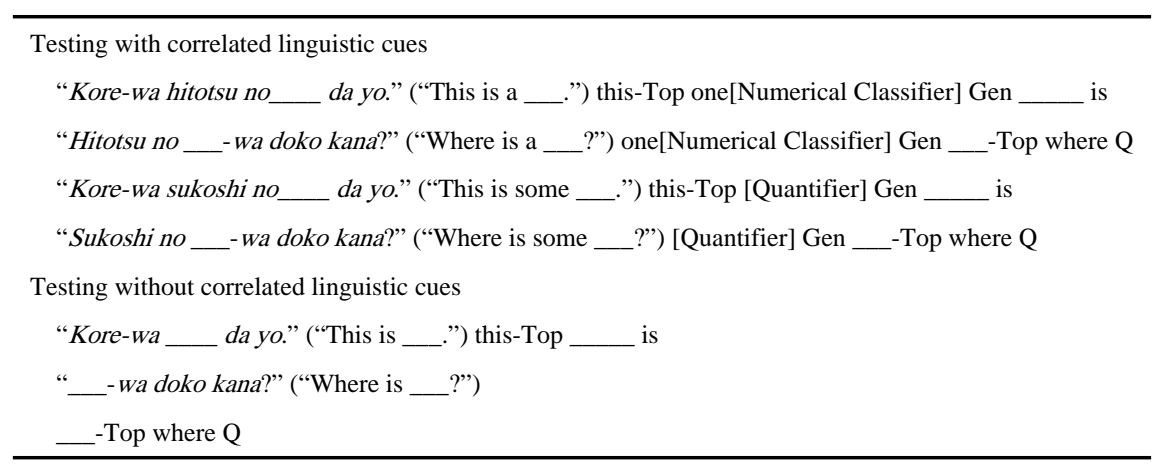

Note. See Table 1 for an explanation of the postpositional particles wa and no.

$\mathrm{Q}=$ uestion marker 
TABLE 3

Proportions of Choices of Each Type of Test Object for Solid and Nonsolid Exemplars

\begin{tabular}{|c|c|c|c|c|}
\hline \multirow[b]{2}{*}{ Exemplar and test object } & \multicolumn{2}{|c|}{$\begin{array}{l}\text { Training with correlated } \\
\text { linguistic cues }\end{array}$} & \multicolumn{2}{|c|}{$\begin{array}{l}\text { Training without correlated } \\
\text { linguistic cues }\end{array}$} \\
\hline & $\begin{array}{l}\text { Test with correlated } \\
\text { linguistic cues }\end{array}$ & $\begin{array}{l}\text { Test without correlated } \\
\text { linguistic cues }\end{array}$ & $\begin{array}{l}\text { Test with correlated } \\
\text { linguistic cues }\end{array}$ & $\begin{array}{l}\text { Test without correlated } \\
\text { linguistic cues }\end{array}$ \\
\hline \multicolumn{5}{|l|}{ Solid exemplar } \\
\hline Shape match & .85 & .82 & .81 & .72 \\
\hline Color match & .07 & .14 & .09 & .13 \\
\hline Material match & .08 & .03 & .14 & .12 \\
\hline \multicolumn{5}{|l|}{ Nonsolid exemplar } \\
\hline Shape match & .08 & .25 & .27 & .32 \\
\hline Color match & .15 & .29 & .43 & .28 \\
\hline Material match & .77 & .46 & .28 & .24 \\
\hline
\end{tabular}

Note. The data for "correct responses" are in boldface. 